\title{
CONHECIMENTOS ESPECIALIZADOS DE PROFESSOR DE BIOLOGIA MOBILIZADOS EM UMA AULA PRÁTICA SOBRE INTERAÇÕES ECOLÓGICAS
}

\author{
CONOCIMIENTOS ESPECIALIZADOS DE PROFESOR DE BIOLOGÍA \\ MOVILIZADOS EN UNA CLASE PRÁCTICA SOBRE INTERACCIONES \\ ECOLÓGICAS
}

Marcela Marques ${ }^{1}$

ORCID iD: 0000-0001-5537-0461

Jeferson Gomes Moriel Junior ${ }^{2}$

ORCID iD: 0000-0003-1526-8002

\begin{abstract}
RESUMO
Uma das implicações acerca dos conhecimentos de professores está em como conduzir aos mesmos, em formação, a integração destes conhecimentos dentro de sua própria prática. Nesse cenário, tem havido um movimento de especialização do conhecimento docente, que se distancia do foco generalista e enfatiza as peculiaridades existentes no ensino das diferentes disciplinas. Para tanto, nosso objetivo é caracterizar o conhecimento especializado mobilizado por professores de Biologia em um relato de aula sobre Interações Ecológicas utilizando o modelo teórico, Biology Teacher's Specialized Knowledge (BTSK) por meio de uma metodologia descritivo-bibliográfica. Com isso, nossos resultados sugerem que nossa práxis docente deve permear entre os conhecimentos pedagógicos e os conhecimentos do conteúdo, caracterizados pelos conhecimentos especializados de professores de Biologia mobilizados durante uma aula prática experimental no Ensino Médio sobre Interações Ecológicas.
\end{abstract}

Palavras-chave: Conhecimento Especializado de Professor. BTSK. Biologia. Interações Ecológicas.

\section{RESUMEN}

Una de las implicaciones sobre el conocimiento de los docentes es cómo llevarlos, en la capacitación, a integrar este conocimiento en su propia práctica. En este escenario, ha habido un movimiento de especialización del conocimiento de la enseñanza, que se distancia del enfoque generalista y enfatiza las peculiaridades existentes en la enseñanza de diferentes disciplinas. Por lo tanto, nuestro objetivo es caracterizar el conocimiento especializado movilizado por los profesores de biología en un informe de clase sobre Interacciones Ecológicas utilizando el modelo teórico, Biology Teacher's Specialized Knowledge (BTSK a través de una metodología descriptiva-bibliográfica. Con esto, nuestros resultados sugieren que nuestra práctica docente debe permear entre el conocimiento pedagógico y el conocimiento del contenido, caracterizado por el conocimiento especializado de los profesores de biología movilizados durante una clase práctica experimental en la Escuela Secundaria de Interacciones Ecológicas.

Palabras clave: Conocimiento Especializado Profesor. BTSK. Biología. Interacciones Ecológicas.

\footnotetext{
${ }_{1}^{1}$ Mestranda, bolsista CAPES no Programa de Pós Graduação em Ensino, Instituto Federal de Mato Grosso (PPGEn IFMT/UNIC ), Cuiabá, Mato Grosso, Brasil. Endereço para correspondência: Antônio Dorileo, 255, 209 C, Coophema, Cuiabá, MT, Brasil, CEP: 78.085-230. E-mail: m.marquesbio@gmail.com.

${ }^{2}$ Doutor em Educação em Ciências e Matemática (UFMT-REAMEC), Docente de Matemática no Instituto Federal de Mato Grosso (IFMT), Cuiabá, Mato Grosso, Brasil. Endereço para correspondência: Profa. Zulmira Canavarros, 95, Centro Norte, Cuiabá, MT, Brasil, CEP: 78048-076. E-mail: jeferson.moriel@ cba.ifmt.edu.br.
} 


\section{INTRODUÇÃO}

Um dos principais problemas da formação de professores não é tanto o desenvolvimento do conhecimento dos alunos, das aulas e da natureza do processo educativo, e sim como facilitar aos professores em formação a integração destes conhecimentos dentro de sua própria prática (CARVALHO, 1993). Corroborando a esse conceito, Furlani (1993) diz que as principais dificuldades encontradas no cotidiano da sala de aula pelos licenciados estão relacionadas à estrutura do curso de formação, ou seja, a inexistência de relação entre as disciplinas durante o processo de formação aliadas à inadequação dos conteúdos das disciplinas universitárias com a realidade do ensino básico.

Nas últimas quatro décadas tem havido uma crescente preocupação de pesquisadores em descrever uma base de conhecimento necessária para ensinar, envolvendo diferentes tipos ou categorias de conhecimentos e dando origem a diversas tipologias do que é necessário para a docência como profissão (SHULMAN, 1986; ALMEIDA; BIAJONE, 2007; RODRIGUES; REALI, 2013). Essas diferentes tipologias têm sido utilizadas tanto para o desenvolvimento de atividades, programas e projetos de formação inicial e continuada de professores, quanto como base teórica para compreender fenômenos associados a preparação docente, a como ensinar e fazer aprender. Neste cenário, tem havido um movimento de especialização do conhecimento docente, que se distancia do foco generalista e enfatiza as peculiaridades existentes no ensino das diferentes disciplinas. Destaca-se como pioneira neste movimento a educação matemática (MORIEL JUNIOR; WIELEWSKI, 2017), ao criar o modelo Mathematical Knowledge for Teaching (BALL; THAMES; PHELPS, 2008) que possuía uma dimensão especializada e, posteriormente, o modelo Mathematics Teacher's Specialized Knowledge - MTSK em que todas as seis dimensões são especializadas (CARRILLO et al., 2014; CARRILLO et al., 2018).

Dado o impacto e reconhecimento do modelo MTSK no mundo (KILPATRICK, SPANGLER, 2015), ele tem sido transposto a outras disciplinas ${ }^{1}$, sendo a Biologia a primeira delas. Isto deu origem ao modelo teórico Biology Teacher's Specialized Knowledge - BTSK (LUÍS, 2015), por meio do qual buscamos neste artigo explorar conhecimentos especializados de Biologia mobilizados em sala de aula para contribuir na compreensão da práxis docente ao ministrar uma aula prática sobre Interações Ecológicas.

\footnotetext{
${ }^{1}$ Para a Física com o modelo Physics Teacher's Specialized Knowledge - PTSK (LIMA, 2018) e para a Química com o modelo Chemistry Teacher's Specialized Knowledge - CTSK (SOARES, 2019), desenvolvidos dentro do TSK Group (Teacher's Specialized Knowlegde Research Group) coordenado pelo Dr. Jeferson Moriel Junio.
} 


\section{CONHECIMENTO ESPECIALIZADO DE PROFESSORES DE BIOLOGIA}

O Biology Teacher's Specialized Knowledge - BTSK (LUÍS, 2015) (Figura 1), utilizado como fundamentador para este estudo, segue o formato hexagonal em consonância com o modelo teórico do Conhecimento Especializado de Professores de Matemática (MTSK) (CARRILLO et al., 2014). O BTSK é dividido em dois domínios: o Conhecimento Didático do Conteúdo (PCK), subdividido em três subdomínios, (i) Conhecimento do Ensino de Biologia (KBT), conhecimento de estratégias metodológicas de ensino da Biologia; (ii) Conhecimento das Características da Aprendizagem da Biologia (KFLB), o conhecimento da forma como os alunos aprendem Biologia por meio utilização de aulas práticas como elemento facilitador; e (iii) Conhecimento dos Standards de Aprendizagem da Biologia (KBLS), conhecimento dos parâmetros de aprendizagem da Biologia, como o currículo, complementos ao currículo, estudos das ações de professores. O outro domínio é o do Conhecimentos da Biologia (BK), também é subdividido em três subdomínios, sendo eles (i) Conhecimento dos Temas da Biologia (KoBT) que é o conhecimento das definições, fenômenos, teorias, leis, da Biologia; (ii) Conhecimento da Estrutura da Biologia (KSB) que é o conhecimento da estrutura da Biologia, e das relações entre os diferentes conteúdos; e (iii) Conhecimento da Natureza da Ciência (KNoS) que aborda sobre o conhecimento da natureza, dos princípios e das grandes ideias (Big Ideas) sobre ciências.

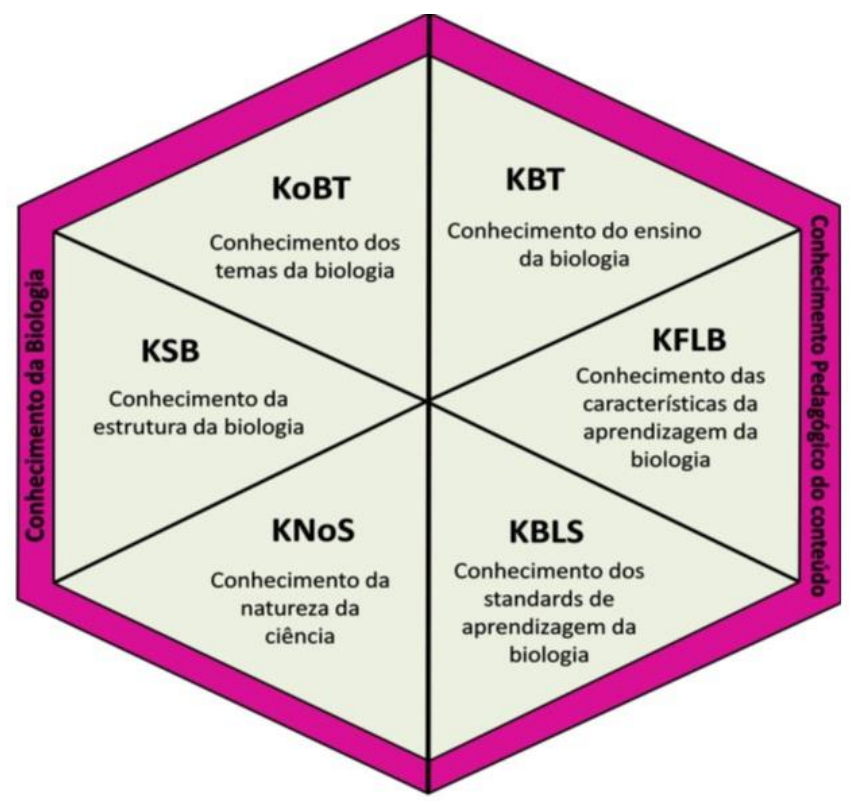

Figura 1 - Modelo de Conhecimento Especializado de Professores de Biologia (BTSK). Fonte: Luís (2015).

Apenas o subdomínio Conhecimentos dos Temas da Biologia (KoBT) tem suas categorias descritas na literatura (LUÍS, 2015) e são apresentadas a seguir: 
- Conceitos e de Exemplos Associados que se referem ao conhecimento das definições ou propriedades específicas, que caracterizam os elementos ou conceitos biológicos e dos exemplos que ajudam a defini-lo;

- Leis, Princípios e Teorias que são os conhecimentos das leis, princípios e teorias associados ao tema;

- Fatos e Fenômenos Biológicos que traz o conhecimento sobre os fatos como verdades dogmáticas e dos fenômenos biológicos enquanto processos e sequências de acontecimentos biológicos;

- Procedimentos e Técnicas de Observação que é o conhecimento sobre os meios e técnicas apropriadas para realizar determinada observação, bem como sobre como e quando fazer;

- Modelos Relacionados com o Conteúdo que retrata sobre o conhecimento sobre estruturas ou registros que permitem diferentes representações de um determinado conteúdo;

- Aplicações do Conteúdo que é sobre a ampla variedade de contextos e aplicações ligada ao conteúdo e que permitem ao professor conhecer os diferentes significados que se podem atribuir ao conteúdo.

Os resultados desse trabalho, e os avanços previstos dessa pesquisa, poderão contribuir com indicadores para descrição de demais categorias para os outros subdomínios do modelo.

\section{METODOLOGIA}

O tipo de pesquisa é qualitativo (BOGDAN; BIKLEN, 1982), de caráter analíticodescritivo sobre conhecimento especializado para ensinar Biologia envolvido em um caso de prática de ensino não tradicional via aula experimental e lúdica.

Quanto ao contexto e fonte de dados, trata-se de uma aula prática experimental lúdica sobre o conteúdo Interações Ecológicas no Ensino Médio, apoiada pelo Programa Institucional de Bolsa de Iniciação à Docência (PIBID), tendo como fonte de dados uma produção escrita em forma de relato de experiência docente fundamentado e publicado por licenciandos (detalhado nos resultados). Os critérios de seleção da amostra foram definidos a partir dos requisitos de Loughran et al. (2001) de classificação de uma produção científica como Professional and Pedagogical Experience Repertoire - PaP-eR (sigla sugerida na publicação original), a saber: ser um relato de prática de ensino de Biologia, estar inseridos em um cenário propício para o ensino, abordar o aspecto prático experimental do ensino de Biologia e permitir a reconstrução do episódio de ensino. O uso de PaP-eR como fonte de dados tem sido adotado por estudos similares (GOES, 
2014; LIMA, 2018; SOARES, 2019; MARQUES; MORIEL JUNIOR, 2019). Por limitações de espaço, neste artigo optamos por explorar apenas uma das seis produções PaP-eR que compuseram o corpus de análise da dissertação em andamento do primeiro autor, sob orientação do segundo.

$A$ obtenção dos dados se deu a partir de leituras sucessivas do PaP-eR selecionado e da extração de trechos que se configurassem como episódios a serem analisados. Os episódios correspondem a um fragmento da produção composto por uma sequência de ações, com sentido completo em si mesmo, com princípio e fim reconhecível, como a execução de uma explicação por exemplo (CARRILLO; ROJAS; FLORES, 2013; MORIEL JUNIOR, 2014).

Para a análise de dados, realizamos a análise de conteúdo (KRIPPENDORFF, 1990), com comparações sistemáticas entre os elementos de informação obtidos nos episódios e a descrição de domínios, subdomínios, categorias e indicadores do marco teórico Biology Teacher's Specialized Knowledge - BTSK. Tais elementos foram classificados em evidências de conhecimentos especializados (trechos que por si só comprovam qual ou quais conhecimentos foram mobilizados pelo professor) ou indícios (trechos que não são suficientes por si só para garantir a existência de um conhecimento, dando oportunidades para o pesquisador seguir investigando para convertê-lo ou não em evidência de conhecimento) (MORIEL JUNIOR; CARRILLO, 2014; FLORES-MENDRANO, 2015).

Utilizamos o instrumento de análise MTSK de Moriel Junior e Alencar (2019), elaborado para originalmente para a educação matemática (Figura 2), e o adaptamos para caracterizar e descrever os conhecimentos especializados de Biologia segundo o BTSK. Os conhecimentos identificados foram codificados com uma sequência alfanumérica indicando o símbolo "§" e o número de identificação do Parágrafo, e a letra "L" seguida da numeração separada por traço para identificar a Linha, sendo que a numeração das linhas é sequencial, iniciando-se a cada parágrafo. Neste trabalho, analisamos um único PaP-eR identificado como P3 no banco de dados da primeira autora e a codificação aqui presente seguirá o seguinte exemplo: P3.§5.L3-7.

\begin{tabular}{||cccc||}
\hline TRECHO DO ARTIGO & & \multicolumn{2}{c||}{ ANÁLISE DO PESQUISADOR } \\
\hline Evidência & Conhecimento... & associado a... & que consiste em... \\
\hline $\begin{array}{c}\text { [Trecho do episódio - linha } \\
\text { ou página, artigo, ano] }\end{array}$ & [subdomínio] & [categoria] & [Síntese do conhecimento] \\
\hline $\begin{array}{c}\text { Exemplo: "eu utilizo a } \\
\text { resolução de problemas para } \\
\text { ensinar derivada" } \\
\text { Artigo, Ano, página) }\end{array}$ & $\begin{array}{c}\text { do ensino de } \\
\text { matemática (KMT) }\end{array}$ & $\begin{array}{c}\text { estratégias de } \\
\text { ensino }\end{array}$ & $\begin{array}{c}\text { uma abordagem de } \\
\text { resolução de problemas } \\
\text { para ensinar derivadas }\end{array}$ \\
\hline
\end{tabular}

Figura 2 - Instrumento de análise MTSK.

Fonte: Moriel Junior e Alencar (2019). 


\section{ANÁLISES E RESULTADOS}

DOI: 10.26571/reamec.v8i2.9747

O PaP-eR - no qual identificamos conhecimentos especializados para ensinar Biologia - relata uma atividade prática intitulada Interações Ecológicas desenvolvida com o objetivo de propiciar a fixação do conteúdo de Interações Ecológicas por parte dos alunos de três turmas de $1^{\circ}$ ano do ensino médio. Os alunos se dividiram em seis grupos e cada grupo ficou sob a supervisão de um bolsista do Programa Institucional de Bolsa de Iniciação à Docência (PIBID). Cada bolsista explanou sobre um tipo de bioma brasileiro para os seus respectivos grupos e, após a explanação, os grupos receberam plaquinhas com nomes de animais de cada bioma, sinais de positivo e negativo, nomes de tipos de interações ecológicas e setas feitas com cartolina e barbante. Com o material em mãos, os alunos deveriam montar uma teia alimentar sendo que as setas deveriam apontar para o animal que seria a presa e os sinais iriam dizer se aquela relação era harmônica ou desarmônica e, ao final da montagem da teia, cada grupo deveria socializar a sua teia com os colegas. (DAMASCENO et al., 2017).

Em síntese, a atividade prática de fixação de conteúdo de Interações Ecológicas pode ser sistematizada nas seguintes etapas:

1. A turma foi dividida em seis grupos e cada bolsista explanou sobre um bioma aos seus respectivos grupos, ficando responsável pela supervisão da atividade prática;

2. Após a explanação, os alunos receberam um envelope com nomes de animais oriundos de cada bioma, setas feitas de cartolina, barbante, sinais de positivo e negativo e plaquinhas com os nomes das relações ecológicos;

3. Os animais deveriam ser organizados de acordo com seus níveis tróficos e o barbante era utilizado para estabelecer a ligação entre eles, indicando pela seta qual animal que se beneficiava com a interação e, em seguida, identificar qual seria a relação entre existente;

4. Ao finalizarem os grupos socializavam suas teias ecológicas e explicava a relação existente entre eles.

Os conhecimentos especializados de professores de Biologia, identificados nesse PaP-eR (Figura 3) sobre Interações Ecológicas, estão dispersos nos subdomínios do modelo BTSK da seguinte maneira: 


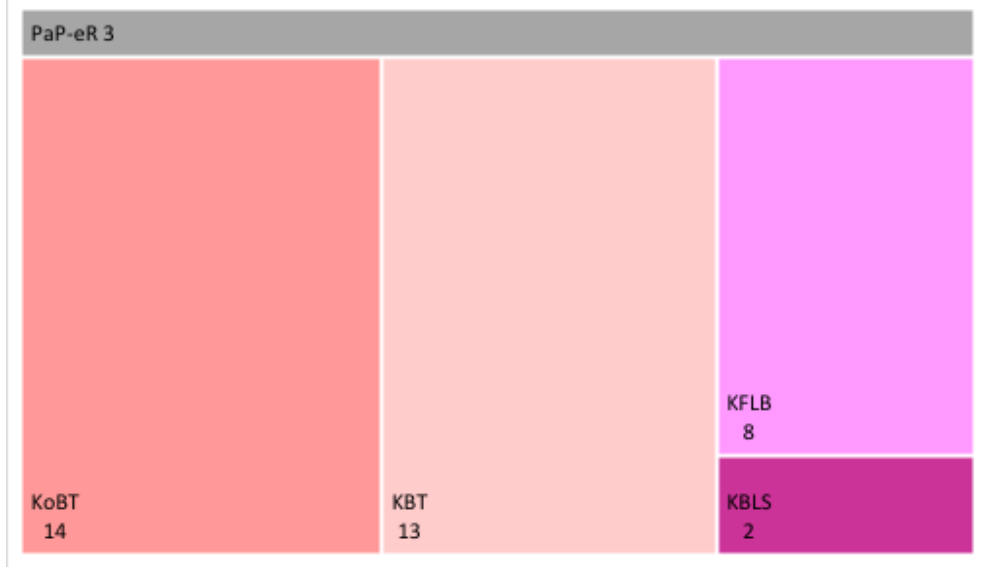

Figura 3 - Conhecimentos Especializados no PaP-eR por Subdomínio do BTSK Fonte: Marques (2020).

Embora a dispersão de conhecimentos seja assimétrica (cf. Figura 3), com prevalência de conhecimentos especializados do domínio pedagógico do conteúdo com a somatória de 23 (vinte e três) frente ao domínio da Biologia com 14 (quatorze) conhecimentos, nota-se a predominância do Conhecimento dos Temas da Biologia - KoBT (14 conhecimentos) mobilizados conforme as estratégias de ensino abordadas na atividade prática lúdica (Conhecimento do Ensino de Biologia - KBT) proposta pelo professor e coordenada pelos pibidianos, como veremos a adiante e explicitados nos Quadros 1 com trinta e duas evidências dos conhecimentos e no Quadro 2 com cinco indícios, totalizando trinta e sete conhecimentos especializados.

\begin{tabular}{|c|c|c|c|}
\hline TRECHO DO ARTIGO & \multicolumn{3}{|c|}{ ANÁLISE DO PESQUISADOR } \\
\hline Manifestação & Conhecimento... & associado a... & que consiste em... \\
\hline $\begin{array}{l}\text { P3.\$3.L1-3: } \\
\text { Zamunaro (2006), muitas } \\
\text { vezes os conteúdos de biologia } \\
\text { são ensinados aos alunos } \\
\text { apenas de maneira teórica } \\
\text { dentro da sala de aula e isso } \\
\text { faz com que estes se } \\
\text { distanciem do assunto, por não } \\
\text { conseguirem contextualizar } \\
\text { com a sua realidade." }\end{array}$ & $\begin{array}{c}\text { do Ensino da } \\
\text { Biologia - KBT }\end{array}$ & $\begin{array}{l}\text { teorias } \\
\text { (in)formais de } \\
\text { ensino }\end{array}$ & $\begin{array}{c}\text { uma característica do } \\
\text { ensino de 'conteúdos de } \\
\text { Biologia': são ensinados } \\
\text { 'apenas de maneira teórica } \\
\text { dentro da sala de aula e } \\
\text { isso faz com que estes se } \\
\text { distanciem do assunto, por } \\
\text { não conseguirem } \\
\text { contextualizar com a sua } \\
\text { realidade.' }\end{array}$ \\
\hline $\begin{array}{l}\text { P3.\$4.L3-6: "Alguns desses } \\
\text { trabalhos como Santos et al. } \\
\text { (2017), Silveira et al. (2016) e } \\
\text { Santos et al. (2015), estão } \\
\text { relacionados a alternativas } \\
\text { como: feiras de ciências, } \\
\text { palestras, debates, jogos } \\
\text { didáticos, júri simulado, aulas } \\
\text { de campo, aulas práticas, }\end{array}$ & $\begin{array}{c}\text { do Ensino da } \\
\text { Biologia - KBT }\end{array}$ & $\begin{array}{c}\text { estratégias de } \\
\text { ensino }\end{array}$ & $\begin{array}{l}\text { exemplos estratégias } \\
\text { alternativas de ensino: } \\
\text { 'feiras de ciências, } \\
\text { palestras, debates, jogos } \\
\text { didáticos, júri simulado, } \\
\text { aulas de campo, aulas } \\
\text { práticas, seminários' }\end{array}$ \\
\hline
\end{tabular}


seminários, entre tantos outros métodos que podem ser utilizados nesse intuito"

P3.\$7.L1-3: "As atividades práticas podem ser aplicadas na abordagem da maioria dos conteúdos de biologia, este trabalho trata, em especial, de uma atividade sobre o conteúdo Interações Ecológicas." do Ensino da

Biologia - KBT estratégias de ensino uma atividade prática de fixação de conteúdo de Interações Ecológicas

P3.\$7.L3-5: As relações ecológicas são interações entre os seres vivos que vivem em um determinado ambiente. Essas interações podem trazer benefícios ou não para os envolvidos, $\mathrm{o}$ que as classificam em harmônicas ou desarmônicas (ARAGUAIA, 2016).

$\begin{array}{ccc}\text { dos Temas da } & \text { conceitos e } & \text { um termo de biologia: } \\ \text { Biologia - KoBT } & \text { exemplos } & \text { interações ecológicas }\end{array}$

$\begin{array}{cc}\begin{array}{c}\text { dos Temas da } \\ \text { Biologia - KoBT }\end{array} & \begin{array}{c}\text { conceitos e } \\ \text { exemplos } \\ \text { associados }\end{array} \\ & \\ & \text { conceitos e } \\ \text { dos Temas da } & \text { exemplos } \\ \text { Biologia - KoBT } & \text { associados }\end{array}$
uma definiçãa das relações
ecológicas

uma propriedade de interações ecológicas: 'podem trazer benefícios ou não para os envolvidos, o que as classificam em harmônicas ou desarmônicas.'

\section{P3.\$8.L1-3: "Segundo} Motokane (2015), é comum que os alunos decorem os nomes das interações ecológicas, e talvez até discutam sobre elas, porém dificilmente conseguem relacionar as suas opiniões com os conceitos científicos aprendidos em sala de aula."

$\begin{array}{cc}\text { das Características } & \text { forma como } \\ \text { da Aprendizagem } & \text { alunos } \\ \text { da Biologia - } & \text { aprendem } \\ \text { KFLB } & \end{array}$

das Características KFLB uma característica da aprendizagem de interações ecológicas: dificuldade de 'relacionar os conceitos científicos aprendidos em sala de aula' com suas 'opiniões'

uma característica de um recurso material (Livros

didáticos aprovados

P3.\$9.L3-6: "Os mesmos [livros] mencionam com ênfase o efeito que uma interação ecológica tem para o indivíduo, mas não tratam da mesma forma o efeito dessa interação em outros níveis de organização, como, por exemplo, para a população, a comunidade ou o ecossistema."

do Ensino da

Biologia - KBT estratégias de ensino

PNLEM 2007/2009): 'não

tratam da mesma forma o efeito dessa interação em outros níveis de organização'

dos Temas da

Biologia - KoBT conceitos e exemplos associados termos da Biologia: 'população' 'comunidade' e 'ecossistema'.
P3.\$20.L1-4: "Durante a preparação da atividade cada bolsista [professor] ficou encarregada de pesquisar sobre um tipo de bioma brasileiro (caatinga, amazônia, cerrado, pampa, mata atlântica e pantanal), suas principais características climáticas, sazonais, de relevo e animais que pertenciam a aquele ambiente." classificações de bioma brasileiro ('caatinga, amazônia, cerrado, pampa, mata atlântica e pantanal') e suas principais características ('climáticas, sazonais, de relevo e animais que pertenciam a aquele ambiente') 
P3.\$22.L1-4: Cada bolsista então, fez uma explanação sobre o bioma do qual ficou responsável para o grupo sob sua supervisão, onde enfatizaram as características marcantes dos mesmos, tais como: onde são encontrados, qual a temperatura média, clima, sazonalidade, relevo, tipo de vegetação, e características do solo.

P3.\$23.L2-5: "Foi entregue aos alunos um envelope contendo plaquinhas feitas em cartolina com nomes de animais oriundos de cada bioma, setas feitas em cartolina e barbante, sinais de mais e menos que representavam positivo e negativo, plaquinhas com nomes de relações ecológicas (predatismo, competição, mutualismo, protocooperação e outros) também feitos em cartolina."

P3.\$24.L1-3: "Os estudantes deveriam organizar as placas com nomes de animais de acordo com o seu nível trófico (primeiro os produtores, depois os consumidores primários, secundários, progressivamente)."

P3.\$24.L3-5: "O barbante estabelece a ligação entre um e outro animal, e a seta deveria mostrar o sentido da relação ecológica, apontando para o animal que se beneficiaria da interação."

\begin{tabular}{|c|c|c|}
\hline $\begin{array}{c}\text { do Ensino da } \\
\text { Biologia - KBT }\end{array}$ & $\begin{array}{c}\text { estratégias de } \\
\text { ensino }\end{array}$ & $\begin{array}{c}\text { uma explicação } \\
\text { instrucional sobre biomas: } \\
\text { expor verbalmente suas } \\
\text { características }\end{array}$ \\
\hline $\begin{array}{c}\text { dos Temas da } \\
\text { Biologia - KoBT }\end{array}$ & $\begin{array}{l}\text { conceitos e } \\
\text { exemplos } \\
\text { associados }\end{array}$ & $\begin{array}{l}\text { características dos biomas: } \\
\text { 'onde são encontrados, } \\
\text { qual a temperatura média, } \\
\text { clima, sazonalidade, } \\
\text { relevo, tipo de vegetação, e } \\
\text { características do solo.' }\end{array}$ \\
\hline $\begin{array}{c}\text { do Ensino da } \\
\text { Biologia - KBT }\end{array}$ & $\begin{array}{c}\text { recurso para } \\
\text { ensinar }\end{array}$ & $\begin{array}{l}\text { características do recurso } \\
\text { material didático lúdico } \\
\text { sobre interações } \\
\text { ecológicas: objetivos e } \\
\text { funções de plaquinhas de } \\
\text { cartolina com nomes de } \\
\text { animais, envelope e } \\
\text { barbante. }\end{array}$ \\
\hline $\begin{array}{c}\text { dos Temas da } \\
\text { Biologia - KoBT }\end{array}$ & $\begin{array}{l}\text { conceitos e } \\
\text { exemplos } \\
\text { associados }\end{array}$ & $\begin{array}{l}\text { classificações de relações } \\
\text { ecológicas: predatismo, } \\
\text { competição, mutualismo, } \\
\text { protocooperação e outros. }\end{array}$ \\
\hline
\end{tabular}

$\begin{array}{ccc}\text { dos Temas da } & \text { conceitos e } & \text { conceitos associados ao } \\ \text { Biologia - KoBT } & \text { exemplos } & \text { nível trófico de animais: } \\ \text { associados } & \text { produtores, consumidores } \\ \text { primários, secundários' }\end{array}$

interação."

$\begin{array}{cc}\text { dos Temas da } & \text { conceitos e } \\ \text { Biologia - KoBT } & \text { exemplos } \\ & \text { associados }\end{array}$

termo de Biologia: relações ecológicas
P3.\$25.L1-2: No meio do barbante que ligava um componente a outro da teia, eles deveriam colocar uma plaquinha com o nome da relação existente ali.

\section{do Ensino da recursos para \\ Biologia - KBT ensinar}

característica da atividade prática lúdica (objetos e funções): o barbante é usado para estabelecer uma relação ecológica entre os animais de um bioma
P3.\$25.L2-4: "Os sinais de positivo e negativo iriam dizer se aquela relação era harmônica (benéfica) ou desarmônica (não benéfica) para cada um dos envolvidos."

$\begin{array}{cc}\text { do Ensino da } & \text { recursos para } \\ \text { Biologia - KBT } & \text { ensinar }\end{array}$

uma explicação instrucional sobre os nomes das relações existentes 
P3.§26.L1-3: "Ao final da montagem, cada grupo foi orientado a socializar a sua teia com os outros grupos, mencionando o bioma ao qual ela pertencia, suas características principais, o nível trófico do qual cada animal fazia parte e o tipo de relação existente entre eles."

\section{das Características \\ da Aprendizagem \\ da Biologia - \\ KFLB \\ forma como os alunos aprendem}

interação entre os alunos como forma alternativa de aprendizado: 'cada grupo foi orientado a socializar'

conceitos dos biomas e definições das principais características e o nível trófico de cada animal: 'o

$\begin{array}{cc}\text { dos Temas da } & \text { conceitos e } \\ \text { Biologia - KoBT } & \text { exemplos } \\ \text { associados }\end{array}$

bioma ao qual ela pertencia, suas características principais, $o$ nível trófico do qual cada animal fazia parte e o tipo de relação existente entre eles'

uma etapa (última) da

do Ensino da estratégia de ensino atividade prática sobre IE: socialização dos resultados (teias) com outros grupos.

P3.\$28.L1-2: "Os alunos interagiram o tempo todo durante a atividade, se mostraram curiosos e interessados em encontrar a melhor forma de montar a teia."
das Características
da Aprendizagem
da Biologia -
KFLB
forma como os alunos
aprendem

uma característica do trabalho prático como integrador e facilitador da aprendizagem

\section{P3.\$29.L1-3: A prática} desenvolvida mostrou-se muito eficiente, pois notamos que a mesma maximizou a aprendizagem que os alunos apresentavam do conteúdo teórico, o que possibilitou a redução da abstração existente com relação aos organismos microscópicos.

P3.§30.L1-3: "Durante a socialização das teias alguns alunos se mostraram mais tímidos, enquanto outros mostraram total domínio tanto do conteúdo, quanto da oratória, demonstrando que conseguiram apreender o que lhes foi passado sobre os biomas brasileiros e sobre as Interações Ecológicas."

P3.\$35.L1-3: "Ao compreender o conteúdo de Interações Ecológicas os alunos apreendem não só conceitos biológicos, mas também sobre sua própria relação como um ser natural com outros seres bióticos." das Características da Aprendizagem

da Biologia -

KFLB teoria (in)formal de aprendizagem uma abordagem de aprendizagem: envolver-se em uma prática maximiza

a 'aprendizagem de conteúdo teórico’ prévio e reduzi a 'abstração existente na relação dos organismos microscópios'

\section{das Características da Aprendizagem da Biologia - KFLB \\ forma como os alunos aprendem}

uma característica potencializadora da aprendizagem por meio da aula prática sobre os biomas e as interações ecológicas uma definição: 'conceitos biológicos’ um nível de desenvolvimento

dos Standards de Aprendizagem KBLS conceitos exemplos associados

parâmetros curriculares conceitual e ontológico (a ser) desenvolvido nessa etapa escolar sobre interações ecológicas: 'conceitos biológicos' e 
noções 'sobre sua própria

relação como um ser

natural com outros seres bióticos.'

P3.\$35.L3-5: "Ele aprende que é impossível um animal ou uma planta sobreviver sem que mantenha nenhuma interrelação com outros indivíduos, e mesmo que essa interação não pareça benéfica para o mesmo, a sua espécie será beneficiada de alguma forma."

P3.\$36.L1-4: "De acordo com os Parâmetros Curriculares Nacionais a capacidade dos alunos de pesquisar, de buscar informações, abalizá-las e selecioná-las, além da capacidade de aprender, criar, formular, ao invés de um simples exercício de memorização, deve propiciar ao mesmo a formular questões, diagnosticar e propor soluções para problemas reais (BRASIL, 1999)."

P3.\$46.L1-4: "Ao final desta prática percebemos que ela foi de suma importância na apreensão do conteúdo por parte dos alunos, pois serviu como um veículo facilitador do processo de ensino e aprendizagem, despertando $o$ interesse dos alunos para o conteúdo Interações Ecológicas que muitos consideram de difícil compreensão."

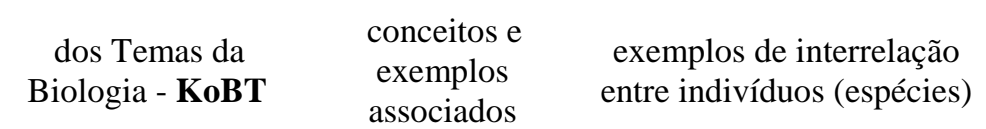

dos Standards de

Aprendizagem da

Biologia - KBLS parâmetros

curriculares expectativa das competências que os alunos devam ter

\section{das Características da Aprendizagem da Biologia - \\ KFLB \\ forma como os alunos aprendem}

uma característica e importância da prática como elementar e motivadora, rompendo bloqueios à aprendizagem

Quadro 1 - Caracterização das Evidências dos Conhecimentos Especializados no relato de prática. Fonte: Damasceno et al. (2017). Elaboração: Marques (2020).

\begin{tabular}{|c|c|c|c|}
\hline TRECHO DO ARTIGO & \multicolumn{3}{|c|}{ ANÁLISE dO PESQUISADOR } \\
\hline Manifestação & Conhecimento... & associado a... & que consiste em... \\
\hline $\begin{array}{l}\text { P3.\$5.L1-3: "A teoria de } \\
\text { Piaget aborda que o ensino } \\
\text { necessita ser acompanhado de } \\
\text { ações e demonstrações e, } \\
\text { sempre que possível, deve } \\
\text { oportunizar atividades práticas } \\
\text { aos alunos } \\
\text { (MOREIRA, 1999)." }\end{array}$ & $\begin{array}{c}\text { do Ensino da } \\
\text { Biologia - KBT }\end{array}$ & $\begin{array}{l}\text { estratégias de } \\
\text { ensino }\end{array}$ & $\begin{array}{l}\text { ações de demonstração: } \\
\text { 'sempre que possível, deve } \\
\text { oportunizar atividades } \\
\text { práticas aos alunos.' }\end{array}$ \\
\hline $\begin{array}{l}\text { P3.\$37.L1-3: "A fim de se } \\
\text { chegar a esse objetivo } \\
\text { consideramos que todas as } \\
\text { formas de abordagem são }\end{array}$ & $\begin{array}{c}\text { das Características } \\
\text { da Aprendizagem } \\
\text { da Biologia - } \\
\text { KFLB } \\
\end{array}$ & $\begin{array}{l}\text { forma como os } \\
\text { alunos aprendem }\end{array}$ & $\begin{array}{c}\text { expectativa de } \\
\text { aprendizagem por meio da } \\
\text { ludicidade }\end{array}$ \\
\hline $\begin{array}{l}\text { válidas, mas as que utilizam a } \\
\text { ludicidade tendem a manter os } \\
\text { estudantes mais concentrados } \\
\text { e interessados." }\end{array}$ & $\begin{array}{l}\text { do Ensino da } \\
\text { Biologia - KBT }\end{array}$ & $\begin{array}{l}\text { estratégias de } \\
\text { ensino }\end{array}$ & $\begin{array}{l}\text { uma característica da } \\
\text { utilização do lúdico como } \\
\text { estratégia de ensino }\end{array}$ \\
\hline
\end{tabular}




\begin{tabular}{|c|c|c|c|}
\hline $\begin{array}{l}\text { P6.\$38.L1-3: } \\
\text { perspectiva Furlan }(2008), \\
\text { salienta que as aulas práticas } \\
\text { tornam-se indispensáveis para } \\
\text { o ensino, pois quando } \\
\text { caracterizadas pela forte } \\
\text { presença do lúdico, auxiliam e } \\
\text { estimulam o interesse e a } \\
\text { participação dos estudantes } \\
\text { nas aulas." }\end{array}$ & $\begin{array}{c}\text { do Ensino da } \\
\text { Biologia - KBT }\end{array}$ & $\begin{array}{l}\text { estratégias de } \\
\text { ensino }\end{array}$ & $\begin{array}{l}\text { uma característica de aulas } \\
\text { práticas lúdicas: 'auxiliam } \\
\text { e estimulam o interesse e a } \\
\text { participação dos estudantes } \\
\text { nas aulas.' }\end{array}$ \\
\hline $\begin{array}{l}\text { P3.\$39.L1-6: "Atividades } \\
\text { lúdicas e práticas quando bem } \\
\text { desenvolvidas normalmente } \\
\text { tem resultados muitos bons, } \\
\text { quando criada e aplicada da } \\
\text { forma correta, é um método de } \\
\text { ensino muito eficiente, e em } \\
\text { alguns estudos reportados na } \\
\text { literatura ressaltam também o } \\
\text { frequente interesse dos alunos } \\
\text { por atividades dessa natureza, } \\
\text { bem como relatos de } \\
\text { professores sobre relevância } \\
\text { da prática experimental na } \\
\text { escola como instrumento para } \\
\text { a aprendizagem de ciências } \\
\text { que somadas à teoria vem } \\
\text { potencializar o processo de } \\
\text { ensino aprendizagem } \\
\text { (FRANCISCO Jr., 2008)." }\end{array}$ & $\begin{array}{c}\text { do Ensino da } \\
\text { Biologia - KBT }\end{array}$ & $\begin{array}{c}\text { estratégias de } \\
\text { ensino }\end{array}$ & $\begin{array}{l}\text { um método de ensino: } \\
\text { 'Atividades lúdicas e } \\
\text { práticas quando bem } \\
\text { desenvolvidas } \\
\text { normalmente tem } \\
\text { resultados muitos bons, } \\
\text { quando criada e aplicada } \\
\text { da forma correta, é um } \\
\text { método de ensino muito } \\
\text { eficiente' }\end{array}$ \\
\hline
\end{tabular}

Quadro 2 - Caracterização dos Indícios dos Conhecimentos Especializados no relato de prática. Fonte: Damasceno et al. (2017). Elaboração: Marques (2020).

No domínio do Conhecimento Pedagógico do Conteúdo (PCK) foram identificados vinte e três conhecimentos, caracterizados em quatorze Conhecimentos do Ensino de Biologia (KBT), oito sobre as Características da Aprendizagem da Biologia (KFLB) e apenas três conhecimentos sobre os Standards de Aprendizagem da Biologia (KBLS); dois deles relatam sobre uma característica do programa PIBID de incentivar estratégias interessantes que auxiliam na aprendizagem (P3.§10.L10-11) e acerca da expectativa das competências que os alunos devem ter previstas nos Parâmetros Curriculares Nacionais 'a capacidade dos alunos de pesquisar, de buscar informações, abalizá-las e selecioná-las, além da capacidade de aprender, criar, formular, ao invés de um simples exercício de memorização, deve propiciar ao mesmo a formular questões, diagnosticar e propor soluções para problemas (P3.§36.L1-4)'. Outra evidência de conhecimento dos Standards de Aprendizagem da Biologia (KBLS) diz a respeito de uma expectativa ontológica manifesta da seguinte forma: 'Ao compreender o conteúdo de Interações Ecológicas os alunos apreendem não só conceitos biológicos, mas também sobre sua própria relação como um ser natural com outros seres bióticos (P3.§35.L1-3)’ que consiste em um nível de desenvolvimento conceitual e ontológico a ser desenvolvido nessa etapa escolar sobre interações ecológicas como 
os conceitos biológicos e as noções sobre sua própria relação como um ser natural com outros seres bióticos', trazendo a importância dos alunos se sentirem pertencentes ao meio ambiente sendo, portanto, integrantes capazes de influenciar a dinâmica de um ecossistema, deixando de ser um telespectador para ser inerente às relações ecológicas. Logo, entende-se Interações Ecológicas como sendo relações entre espécies que vivem numa comunidade; especificamente é o efeito que um indivíduo de uma espécie pode exercer sobre um indivíduo de outra espécie (ACIESP, 1997, p. 148 apud QUESADO, 2009).

\begin{abstract}
$\mathrm{O}$ entendimento dos diferentes fenômenos que englobam essas relações e interações entre seres vivos (incluindo o homem) e os componentes abióticos é amplamente discutido à luz de teorias ecológicas. O ambiente é alterado, físico e quimicamente, pela maneira com os indivíduos realizam suas atividades. Também as interações entre organismos, têm influência na vida de outros seres, da mesma espécie e de espécies diferentes (BEGON, 2007, p. 223).
\end{abstract}

Partindo dessa sustentação do Conhecimento dos Standards de Aprendizagem da Biologia (KBLS), ancoram-se os outros dois conhecimentos dos subdomínios do Conhecimento Pedagógico do Conteúdo (PCK) sobre o ensino e a aprendizagem, fundamentando as atividades práticas e atividades lúdicas e o papel dessas no processo de aprendizagem, de interação e de pertencimento ao conteúdo ministrado.

Para descrever as etapas da atividade prática lúdica, o relato inicia-se fundamentando a prática com referências teóricas caracterizadas como o Conhecimento do Ensino da Biologia (KBT), abordando sobre as alternativas de estratégias de aulas práticas como: 'feiras de ciências, palestras, debates, jogos didáticos, júri simulado, aulas de campo, aulas práticas, seminários, entre tantos outros métodos que podem ser utilizados nesse intuito', sobre a fragilidades do ensino: 'são ensinados aos alunos apenas de maneira teórica dentro da sala de aula e isso faz com que estes se distanciem do assunto, por não conseguirem contextualizar com a sua realidade'. Esse conhecimento especializado se fez presente em longo de todo artigo, na introdução, nas etapas da metodologia (aplicação da atividade prática lúdica) e nos resultados e discussão, fortalecendo a estratégia de ensino como a exposição verbal para explicar as características dos biomas (P3.§22.L1-4) e explorando a uma característica do recurso lúdico sobre interações ecológicas com plaquinhas de cartolina, envelope e barbante, visando maximizar a aprendizagem (P3.§29.L1-3), orientando os alunos a socializarem os resultados (P3.§26.L1-3).

Como consolidação à prática lúdica da estratégia de ensino, o Conhecimento das Características da Aprendizagem da Biologia (KFLB) faz menção à uma fragilidade da aprendizagem fundamentada na seguinte manifestação: 'Segundo Motokane (2015), é comum que os alunos decorem os nomes das interações ecológicas, e talvez até discutam sobre elas, porém dificilmente conseguem relacionar as suas opiniões com os conceitos científicos aprendidos em 
sala de aula' (P3.§8.L1-3). Portanto, após a realização da atividade prática, vemos nas manifestações as constatações de que os alunos romperam esse bloqueio à aprendizagem: 'Ao final desta prática, percebemos que ela foi de suma importância na apreensão do conteúdo por parte dos alunos, pois serviu como um veículo facilitador do processo de ensino e aprendizagem, despertando o interesse dos alunos para o conteúdo Interações Ecológicas que muitos consideram de difícil compreensão', pois a 'aula prática facilitou à aprendizagem, reduzindo a abstração existente na relação dos organismos microscópios', proporcionando a interação entre os alunos, como apresentado por Cherato (2014):

\begin{abstract}
A aprendizagem depende das características pessoais, do ritmo, das motivações e interesses individuais, assim, a forma mais adequada de ensino a ser utilizada tende a variar segundo as necessidades do aluno. Aprender ecologia na escola permite ao aluno compreender a forma que o ser humano se relaciona com a natureza além de ampliar seu entendimento sobre o mundo vivo (PURVES, 2005, p.1044 apud CHERATO, 2014).
\end{abstract}

No domínio do Conhecimento de Biologia (BK), apenas o subdomínio do Conhecimento dos Temas da Biologia (KoBT) foi identificado, sendo um conhecimento na categoria de Aplicações do Conteúdo, que consistem em uma característica de um recurso material (Livros didáticos aprovados Programa Nacional do Livro Didático para o Ensino Médio - PNLEM 2007/2009): 'não tratam da mesma forma o efeito dessa interação em outros níveis de organização', e treze conhecimentos na categoria de Conceitos e Exemplos Associados em que se percebe por parte dos licenciandos bolsistas a abordagem acerca do conteúdo no que diz respeito a conceitos, definições e exemplos, como pode-se ver, por exemplo, nas seguintes manifestações: 'Cada bolsista então, fez uma explanação sobre o bioma do qual ficou responsável para o grupo sob sua supervisão, onde enfatizaram as características marcantes dos mesmos, tais como: onde são encontrados, qual a temperatura média, clima, sazonalidade, relevo, tipo de vegetação, e características do solo'; 'Ele aprende que é impossível um animal ou uma planta sobreviver sem que mantenha nenhuma interrelação com outros indivíduos, e mesmo que essa interação não pareça benéfica para o mesmo, a sua espécie será beneficiada de alguma forma'.

Esse subdomínio do Conhecimento dos Temas da Biologia (KoBT) não foi apenas o único a ser identificado no domínio do Conhecimento de Biologia (BK), mas foi o conhecimento que esteve presente em todas as etapas da instrução da atividade prática lúdica, como exemplificado a seguir:

- Etapa 1 - Preparação da atividade (P3.§20.L1-4): quando os bolsistas fizeram as classificações de bioma brasileiro "(caatinga, amazônia, cerrado, pampa, mata atlântica e pantanal" e apresentaram as principais características ("climáticas, sazonais, de relevo e animais que pertenciam a aquele ambiente"); e 'onde são encontrados, qual a temperatura 
média, clima, sazonalidade, relevo, tipo de vegetação, e características do solo' (P3.§22.L1-4);

- Etapa 2 - Entrega do material lúdico com seus significados (P3.§23.L2-5), que consiste em classificações de relações ecológicas: predatismo, competição, mutualismo, protocooperação e outros, e 'nos conceitos que definem o nível trófico de cada animal' (P3.§24.L1-3);

- Etapa 3 - Instrução sobre os sinais de positivo e negativo, que consiste no conceito de relações interespecíficas das espécies 'relação era harmônica (benéfica) ou desarmônica (não benéfica)' (P3.§25.L2-4). Fundamentados na literatura como:

Os diferentes tipos de interações, harmônicas e desarmônicas, podem ainda ser separadas em intraespecíficas, quando ocorrem entre organismos da mesma espécie, e interespecíficas, quando os organismos envolvidos na interação são de espécies distintas. (LOPES, 2010, p. 145 apud CHERATO, 2014).

Nas relações harmônicas há benefício mútuo entre os grupos de espécies envolvidos, ou beneficio para um dos organismos, sem prejuízo para o outro. Já as relações desarmônicas ou negativas são aquelas nas quais há prejuízo para algum dos grupos de espécies envolvidos, com benefício do outro (MACHADO, 2003, p. 484 apud CHERATO, 2014).

Nas relações interespecíficas desarmônicas, se inclui o amensalismo em que um ser vivo produz e libera compostos químicos que inibem ou impedem a sobrevivência do outro; o predatismo onde um indivíduo mata outro para se alimentar; o esclavagismo, que consiste na exploração de uma espécie por outro o parasitismo no qual o parasita retira, do corpo do hospedeiro, nutrientes para garantir a sua sobrevivência, e por ser prolongada acaba debilitando-o; competição que é a disputa por alimento ou espaço, entre indivíduos e espécies diferentes (BEGON et al., 2007, 234).

- Etapa 4 - Final da montagem da teia em que os alunos eram orientados a socializarem suas teias com os outros grupos (P3.§26.L1-3), que consiste nos conceitos dos biomas e definições das principais características e o nível trófico de cada animal: 'mencionando o bioma ao qual ela pertencia, suas características principais, o nível trófico do qual cada animal fazia parte e o tipo de relação existente entre eles'.

Além das evidências dos conhecimentos especializados, identificamos cinco indícios que, por se tratar de PaP-eR, a continuidade na investigação quanto à sua caracterização fica limitada pelo fato de não termos como aprofundar nos significados intrínsecos nos trechos do relato. Nesse caso, os indícios tratam de conceitos generalistas sobre a efetividade, característica e expectativas da atividade lúdica que, ao ser analisada no contexto do PaP-eR, permite-nos apontá-los como evidências desde que fundamentadas em outros trechos que consistem em uma característica do trabalho prático como integrador e facilitador da aprendizagem sobre os biomas e as interações ecológicas. 


\section{CONSIDERAÇÕES}

Com este trabalho, conseguimos além de caracterizar o conhecimento especializado de professores de Biologia sobre Interações Ecológica, compreender como as relações entre esses conhecimentos fundamentam o conhecimento especializado que o professor de Biologia mobilizou durante a aula, em que, para tanto, o mesmo deve dispor de conhecimentos pedagógicos sobre estratégias de ensino e de fortalezas de aprendizagem, envolvendo também o conhecimento de parâmetros curriculares.

O Indicador de Adequação da Formação Docente, que apresenta a relação entre a formação inicial de professores de uma escola e as disciplinas por estes lecionadas, demostra que para nenhuma disciplina do ensino médio este índice atinge $80 \%$ de adequação, apresentando a disciplina de Biologia o melhor percentual que é de 79,4\%. (BRASIL, 2017). Porém, mesmo a disciplina da Biologia estando em destaque no ranking, não podemos deixar de mencionar que é considerável o percentual de $20,6 \%$ de que não possuem formação superior em licenciatura, então tratar desses conhecimentos na licenciatura apode ajudar a melhor esses índices a médio prazo.

Acreditamos que a não contemplação dos subdomínios do Conhecimento da Natureza da Ciência $(\mathrm{KNoS})$ e do Conhecimento da Estrutura da Biologia (KSB) se deve à maneira como o conteúdo foi abordado e descrito no PaP-eR, o que não configura uma fragilidade metodológica na caracterização do conhecimento especializado. Contudo, notamos que são necessárias mais pesquisas para avançar na compreensão do papel destes subdomínios na prática docente.

A caracterização dos conhecimentos expostos nos resultados nos permite concluir que o conhecimento especializado se dá na relação entre os subdomínios que nos dizem sobre o ponto chave mobilizado em aula para o acontecimento do processo de ensino-aprendizado do ensino de Biologia. Sendo assim, corroborando com a legitimidade do modelo transposto para Biologia como uma ferramenta para compreensão e melhoria da formação de professores. Estes resultados reforçam a importância da continuidade das pesquisas que utilizem e contribuíam para o desenvolvimento do BTSK.

A expectativa é que continuemos a desenvolver pesquisas com o modelo teórico BTSK, incluindo metodologias tanto teóricas (como deste artigo), quanto empíricas, utilizando as gravações de aulas. É importante avançarmos também na compreensão das conexões entre os conhecimentos.

Consentimos que, com profissionais mais capacitados, dotados de conhecimentos especializados, haja melhoria da qualidade do ensino da Biologia, progresso na atuação da profissão docente e da sua valorização. 


\section{AGRADECIMENTOS}

Os autores agradecem o apoio da Coordenação de Aperfeiçoamento de Pessoal de Nível Superior

- Brasil (CAPES) - Código de Financiamento 001, da FAPEMAT (Edital Universal 42/2016/FAPEMAT) e do IFMT (Chamadas 04/2018 e 01/2019/DPG/PROPES/IFMT).

\section{REFERÊNCIAS}

ALMEIDA, P. C. A.; BIAJONE, J. Saberes docentes e formação inicial de professores: implicações e desafios para as propostas de formação. Educação e pesquisa, v. 33, n. 2, p. 281 $295,2007$.

BALL, D. L.; THAMES, M. H.; PHELPS, G. Content Knowledge for Teaching: What Makes It Special? Journal of teacher education, v. 59, n. 5, p. 389-407, 2008.

BEGON, M.; COLIN R.; TOWNSENDE, J.; HASPER L..Tradução Adriano Sanches Ecologia de Indivíduos a Ecossistemas. $4^{\text {a }}$ Edição. Artmed Editora. Porto Alegre, 2007.

BOGDAN, R. C.; BIRTEN, S. K. Qualitative research for education; an introduction for to theory and methods. Boston, .Allyn and Bacon, p. 27-30, 1982.

BRASIL. Ministério da Educação. Instituto Nacional de Estudos e Pesquisas Educacionais Anísio Teixeira - INEP (Comp.). Censo escolar da educação básica 2016: Notas estatísticas. Brasília. p 29. 2017.

CARRILLO, J., CLIMENT, N., CONTREARAS, L.C., MONTES, M.A., ESCURO-ÁVILA, D.; FLORES-MEDRANO, E. (Eds.). Un marco teórico para el conocimiento especializado del profesor de Matemáticas. Huelva: Universidad de Huelva Publicaciones, 2014.

CARRILLO, J., CLIMENT, N., MONTES, M., CONTRERAS, L.C., FLORES-MEDRANO, E., ESCUDERO-ÁVILA, D., VASCO, D., ROJAS, N., FLORES, P., AGUILAR-GONZÁLEZ, A., RIBEIRO, M.; MUÑOZ-CATALÁN, M.C. The Mathematics Teacher's Specialised Knowledge (MTSK) model. Research in Mathematics Education, v. 20, n. 3, 236-253, 2018.

CARVALHO, A. M. P. Reformas nas licenciaturas: a necessidade de uma mudança curricular. Instituto Nacional de Estudos Pedagógicos, n. 54, p. 51-64, 1993.

CHERATO, J. T. Relações Ecológicas entre es Seres Vivos: da Teoria à Prática. Produção Didático Pedagógica desenvolvida ao programa de Desenvolvimento Educacional -PDE SEED/PR, Universidade Estadual do Centro- Oeste do Paraná / UNICENTRO. Paraná, v. 2, 2014.

DAMASCENO, I. T.; GUIMARÃES, M. S.; PEREIRA, N. A.; ALMEIDA, O. da S. Abordagem do Conteúdo Interações Ecológicas no Contexto do PIBID: utilizando uma atividade prática como ferramenta facilitadora no processo de ensino e aprendizagem. In: Seminário GEPRÁXIS VI Seminário Nacional e II Seminário Internacional Políticas Públicas, Gestão e Práxis Educacional, 2017, Vitótia da Conquista, BA. Anais... Seminário GEPRÁXIS, Vitótia da Conquista, BA 6 (6), 1661-1672, 2017. Disponível em: http://anais.uesb.br/index.php/semgepraxis/issue/current. Acesso em 05 de Dezembro de 2019. 
FLORES-MEDRANO, E; ESCUDERO-ÁVILA, D. I; MONTES, M; AGUILAR, A; CARRILLO, J. Nuestra Modelación del Conocimiento Especializado del Profesor de Matemáticas, el MTSK. In: CARRILLO, J. et al. (Org.). Un Marco teórico para el Conocimiento Especializado del Profesor de Matemáticas. Huelva: Universidad de Huelva Publicaciones, p. 70-92, 2014.

FLORES-MENDRANO, E. Una profundización en la conceptualización de elementos del modelo de Conocimiento Especializado del Profesor de Matemáticas (MTSK). 2015. Tesis doctoral. Univesidad de Huelva, Espanha.

FURLANI, J. A Formação do Professor de Biologia no curso de Ciências Biológicas da Universidade Federal de Santa Catarina: uma contribuição à reflexão. Dissertação de Mestrado. Florianópolis: Centro de Ciências da Educação - Universidade Federal de Santa Catarina, 1993.

GOES, L F. de. Conhecimento Pedagógico do conteúdo: estado da arte no campo da educação e no ensino de química. 2014. 155 f. Dissertação (Mestrado em Ensino de Química) Universidade de São Paulo - USP, São Paulo.

KILPATRICK, J.; SPANGLER, D. A. Educating Future Mathematics Education Professors. Handbook of International Research in Mathematics Education, p. 297, 2015.

LIMA, S. S, COSTA, L. D., SOARES, S. T. C, SILVA FILHO, V. P., MORIEL JUNIOR, J. G. e MELLO, G. J. Análise de PaP-eRs como primeira aproximação metodológica para configurar o modelo de conhecimento especializado de professores de física (PTSK). In: Congresso Internacional de Formação e Desenvolvimento Profissional Docente - Residência Docente: Paradigma de Integração Teoria-Prática, n. 3, p. 1-5, 2017.

LIMA, S. S. Conhecimento Especializado de Professores de Física: Uma proposta de Modelo Teórico. 2018. 144 f. Dissertação (Mestrado em Ensino). Programa de Pós-graduação em Ensino, Instituto Federal de Educação, Ciências e Tecnologias do Estado de Mato Grosso, Cuiabá.

LUÍS, M. Conhecimento Especializado de Professores de Biologia. 2015. Tese (Doutorado). Univesidad de Huelva, Espanha (Documento Inédito).

LOUGHRAN, J., MILORY, P., BERRY, A., GUNSTONE, R. e MULHALL, P. Documenting science teachers' pedagogical content knowledge through PaP-eRs. Research in Science Education, v. 31, n. 2, p. 289-307, 2001.

MARQUES, M.; MORIEL JUNIOR, J. G. Conhecimento Especializado de Professores de Biologia: uma Análise de Pap-eR sobre Embriologia Humana. In: CONGRESO IBEROAMERICANO SOBRE CONOCIMIENTO ESPECIALIZADO DEL PROFESOR DE MATEMÁTICAS. Huelva: CGSE. 2019.

MARQUES, M. Conhecimento Especializado de Professores de Biologia: análise de relatos de prática no Ensino Médio. 2020. 106 f. Dissertação (Mestrado em Ensino). Programa de Pósgraduação em Ensino, Instituto Federal de Educação, Ciências e Tecnologias do Estado de Mato Grosso, Cuiabá, (Documento Inédito). 
MORIEL JUNIOR, J. G.; ALENCAR, A. P. Conhecimento especializado para ensinar Cálculo: um panorama da produção do COBENGE 2012-2017. Brazilian Journal of Development, v. 5, n. 7, p. 7687-7702, 2019.

MORIEL JUNIOR, J. G.; WIELEWSKI, G. D. Base de conhecimento de professores de matemática: do genérico ao especializado. Revista de Ensino, Educação e Ciências Humanas, v. 18, n. 2, p. 126-133, 2017.

QUESADO, L. B. Interações Ecológicas nos Livros Didáticos do Ensino Médio.2009. p. 114. Instituto de Biologia Roberto Alcântara Gomes da Universidade do Estado do Rio de Janeiro (Monografia em Ciências Biológicas). Rio de Janeiro.

RODRIGUES, M. da C. C; REALI, A. M. de M. R. Reflexões sobre a base de conhecimento para o exercício da profissão docente: narrativas das professoras colaboradoras de um programa de formação continuada on-line. Revista de Educação Pública, Cuiabá, MT, v. 22, n. 50, p. 643-663, 2013.

SHULMAN, L. Those who understand: Knowledge growth in teaching. Educational researcher, v. 15, n. 2, p. 4-14, 1986.

Knowledge and teaching: Foundations of the new reform. Harvard educational review, v. 57, n. 1, p. 1-23, 1987.

SOARES, S. T. C. Conhecimento Especializado de Professores de Química - CTSK:

Proposta de Modelo Teórico. 2019. 88 f. Dissertação (Mestrado em Ensino). Programa de PósGraduação Stricto Sensu em Ensino, Instituto Federal de Educação, Ciência e Tecnologia de Mato Grosso, Cuiabá.

Submetido em: 30 de janeiro de 2020. Aprovado em: 26 de fevereiro de 2020. 